\title{
Dietary intakes of children aged between 1-3 years attending a childcare centre in Dublin
}

\author{
M. C. Walsh ${ }^{1}$, J. L. O’Neill ${ }^{1}$, J. O’Dea ${ }^{1}$, E. R. Gibney ${ }^{2}$, A. Regan ${ }^{1}$ and E. Shannon ${ }^{1}$ \\ ${ }^{1}$ Danone Baby Nutrition, Block 1 Deansgrange Business Park, Deansgrange, Co. Dublin, Republic of Ireland and \\ ${ }^{2}$ UCD Institute of Food and Health, University College Dublin, Belfield, Dublin 4, Republic of Ireland
}

The period between the ages 1-3 years is one of rapid growth and appropriate dietary intake is therefore required to provide the nutrients to support optimum growth and development ${ }^{(1)}$.

The aim of this study was to calculate the nutrient intakes of 40 healthy pre-school children aged 1-3 years and assess whether they met the recommended intakes for energy and key nutrients ${ }^{(2-5)}$. This was done by identifying individuals meeting the recommended $\operatorname{targets}^{(2-5)}$ to allow for individual feedback to be generated. Parents/guardians provided written consent for their child to take part in the study. Food and beverage intake was recorded by parents/guardians/staff as appropriate, using a 3-day food diary. Questionnaires were also administered to parents/guardians to provide health, lifestyle and attitudinal information. Dietary intake data was analysed using Dietplan 6 (Forestfield Software Ltd, UK) ${ }^{(6)}$.

\begin{tabular}{|c|c|c|c|c|c|}
\hline \multirow[b]{2}{*}{$(n=40)$} & \multirow[b]{2}{*}{ RDA } & \multirow[b]{2}{*}{ Mean } & \multirow[b]{2}{*}{ SD } & \multicolumn{2}{|c|}{$\%$ Toddlers (Individual level) } \\
\hline & & & & Meeting RDA & Not Meeting RDA \\
\hline \multicolumn{6}{|l|}{ Energy and Macronutrients } \\
\hline Energy (kcal) & $1197.5 *$ & 1198.0 & 242.4 & 43 & 58 \\
\hline Protein $(g)$ & $14.5^{*}$ & 47.3 & 7.9 & 100 & 0 \\
\hline$\%$ Energy from Carbohydrate & $50 *$ & 47.1 & 6.1 & 35 & 65 \\
\hline$\%$ Energy from Fat & $35^{*}$ & 34.1 & 5.2 & 70 & 30 \\
\hline$\%$ Energy from Saturated Fat & $11^{*}$ & 15.9 & 2.8 & 8 & 93 \\
\hline \multicolumn{6}{|l|}{ Vitaminŝ } \\
\hline Vitamin A (ug RE) & $400 \dagger$ & 483.3 & 171.1 & 63 & 38 \\
\hline Vitamin D (ug) & $15 \ddagger$ & 3.1 & 3.2 & 0 & 100 \\
\hline Thiamin (mg) & $0.5 \dagger$ & 1.0 & 0.2 & 100 & 0 \\
\hline Vitamin $\mathrm{B}_{12}$ (ug) & $0.7 \dagger$ & 6.2 & 5.7 & 100 & 0 \\
\hline Vitamin $\mathrm{B}_{6}(\mathrm{mg})$ & $0.7 \dagger$ & 1.2 & 0.3 & 95 & 5 \\
\hline Riboflavin (mg) & $0.8 \dagger$ & 1.7 & 0.4 & 100 & 0 \\
\hline Vitamin C (mg) & $45 \dagger$ & 67.5 & 34.9 & 73 & 28 \\
\hline \multicolumn{6}{|l|}{ Mineralŝ } \\
\hline Calcium (mg) & $700 \ddagger$ & 860.3 & 231.6 & 75 & 25 \\
\hline Iron (mg) & $8 \dagger$ & 8.5 & 3.3 & 50 & 50 \\
\hline Sodium (mg) & 800 & 1251.6 & 337.4 & 5 & 95 \\
\hline Zinc (mg) & $4 \dagger$ & 5.7 & 1.6 & 88 & 13 \\
\hline Iodine (ug) & $70 \dagger$ & 158.4 & 57.8 & 95 & 5 \\
\hline
\end{tabular}

Although the majority of parents $(84 \%)$ reported that their child had a healthy diet, a large proportion of toddlers did not meet their recommended intake for energy or key macro- or micronutrients ${ }^{(2-5)}$. This study indicates a lack of understanding about healthy eating and the specific nutritional requirements of children aged 1-3 years.

1. Lanigan J, Turnbull B, Singhal A (2007) Toddler diets in the UK: deficiencies and imbalances. 2. Relationship J Fam Health Care 17(6): 197-200.

2. Department of Health. Dietary Reference Values for Food Energy and Nutrients for the United Kingdom. London: HMSO, 1996.

3. Food Safety Authority of Ireland. Recommended Dietary Allowances for Ireland. Dublin:FSAI, 1999. Available from: www.fsai.ie

4. Ross CA, Taylor CL, Yaktine AL et al. editors. Dietary reference intakes for Calcium and Vitamin D. Washington, D.C.: Institute of Medicine, 2011. Available from: www.nap.edu

5. NHS choices. Salt: the facts [Internet]. UK:NHS, 2011 [cited 2011 July]. Available from: www.nhs.uk/Livewell/Goodfood/Pages/salt.aspx

6. Food Standards Agency. McCance and Widdowson's The Composition of Foods, $6^{\text {th }}$ Edition. Cambridge:Royal Society of Chemistry, 2002. 